\title{
A systematic evaluation of the compatibility of histones containing methyl-lysine analogues with biochemical reactions
}

Cell Research (2009) 19:1217-1220. doi: 10.1038/cr.2009.110; published online 22 September 2009

\section{Dear Editor,}

Histone lysine methylation has received a great deal of attention from the chromatin field over the past 10 years. To date, histone lysine methylations have been demonstrated to play pivotal roles in nearly all biological processes involving chromatin, including replication, transcription, DNA repair etc. [1]. One major challenge in the field has been that of obtaining homogenously methylated histones, i.e., histones methylated at a specific lysine residue with a particular methyl status. Such histone materials could be of important use in many functional applications. In 2007, Shokat's lab invented an easy, robust alkylating reaction (Shokat's reaction) that chemically installs specific methyl-lysine analogues (MLAs) onto specific histone residues [2]. It is a very promising technology that may revolutionise our understanding of methylated histone lysine-mediated biological functions. However, Shokat's reaction is yet to be widely adopted by biologists. Some researchers may worry about the compatibility of the "pseudo lysine" generated by Shokat's reaction with subsequent biochemical assays. Shokat's reaction product carries an $N$-methylated aminoethylcysteine (Kc or "pseudo lysine"), which replaces the $\gamma$-methylene with a sulphide, resulting in a slight lengthening of the side chain $(0.28$ $\AA$ ) and a small increase in acidity ( $-1.1 \mathrm{pKa}$ unit) [2]. Here, we report a systematic evaluation of the products of Shokat's reaction in various biochemical assays, using different classes of methylated histone-binding proteins, histone lysine methyltransferases (HKMTs) and histone demethylases.

Recognition of methylated histones by effector proteins (or "readers") is critical for the biological function of histone methylation. Thus, it is important to test whether the artificially "methylated" histones generated by MLA technology can still be recognised by their concomitant methylated histone-binding proteins.
In the initial report of MLA technology, HP1 $\alpha$ displayed binding activity towards $\mathrm{H} 3 \mathrm{Kc} 9 \mathrm{me} 2$ (H3 with its amino acid No. 9 converted to $N$-dimethyl-aminoethylcysteine or "pseudo" dimethyl-lysine) [2]. We repeated this experiment with all four methyl statuses (me0/1/2/3). Indeed, HP1 $\alpha$ bound "methylated" $\mathrm{H} 3 \mathrm{Kc} 9$, but not its unmethylated form (Figure 1A, upper panel). Moreover, it preferentially bound the trimethyl and dimethyl forms (Figure 1A, upper panel), which is a characteristic feature of HP1's interaction with native methylated H3K9. In a competition experiment, both H3K9me3 and $\mathrm{H} 3 \mathrm{Kc} 9 \mathrm{me} 3$ peptides were capable of eluting HP1 $\alpha$ protein bound by biotin-labelled $\mathrm{H} 3 \mathrm{Kc} 9 \mathrm{me} 3$ peptide at similar concentrations (Figure 1A, lower panel).

We then tested other domains that recognise methylated histones. 53BP1, the mammalian homologue of the fission yeast protein $\mathrm{Crb} 2$, specifically binds methylated H4K20 through its tudor domain [3]. Interestingly, 53BP 1 cannot bind H4K20me 3 due to structural hindrance [3]. In our peptide pull-down assay, recombinant 53BP1 tudor domain recognised MLAgenerated $\mathrm{H} 4 \mathrm{Kc} 20 \mathrm{me} 1$ and $\mathrm{H} 4 \mathrm{Kc} 20 \mathrm{me} 2$, but not the trimethyl or unmethylated forms (Figure 1B, upper panel). In a competition experiment, a slightly higher concentration of $\mathrm{H} 4 \mathrm{Kc} 20 \mathrm{me} 2$ peptide was required to elute bound 53BP1 (Figure 1B, lower panel), suggesting that 53BP1 has slightly lower binding affinity towards H4Kc20me2 peptide compared with the authentic H4K20me2 peptide. Recently, G9a and GLP were demonstrated to bind methylated H3K9 through a new methylated histone recognition domain, ankyrin repeats [4]. We thus analysed the G9a ankyrin repeats in the peptide pull-down assay. $\mathrm{H} 3 \mathrm{Kc} 9 \mathrm{me} 1$ and $\mathrm{H} 3 \mathrm{Kc} 9 \mathrm{me} 2$ were capable of being recognised by the recombinant G9a ankyrin repeats, but not by the trimethyl or unmethylated forms (Figure 1C, upper panel). This methyl status specificity is consistent with the previous report on native methylated peptides [4]. In addition, 
G9a ankyrin repeats displayed comparable binding affinity towards $\mathrm{H} 3 \mathrm{~K} 9 \mathrm{mel}$ and $\mathrm{H} 3 \mathrm{Kc} 9 \mathrm{mel}$ peptides in a competition experiment (Figure 1C, lower panel).

The above experiments demonstrated the compatibility of MLA products with methylated histonebinding proteins in binding assays that do not involve any enzymatic reaction. Therefore, we extended our evaluation of MLA products to enzymatic reactions that directly use methylated histones as substrates. In the initial report of MLA technology, the founding member of the HKMT family, Suv39h1, displayed comparable activity towards MLA-generated H3 tail peptide versus
A

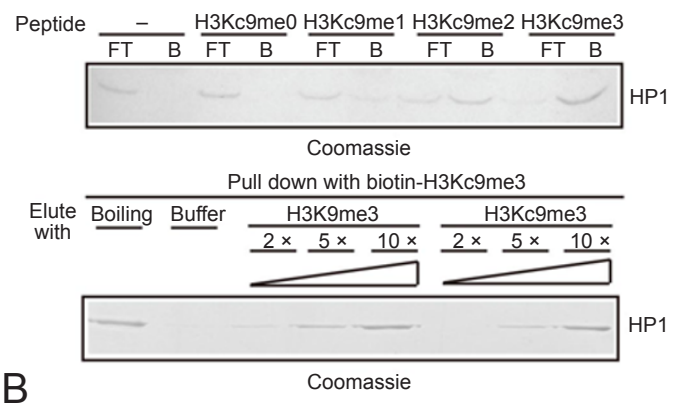

Peptide _ _ $\mathrm{H} 4 \mathrm{Kc20me0}$ H4Kc20me1 $\mathrm{H} 4 \mathrm{Kc} 20 \mathrm{me} 2 \mathrm{H} 4 \mathrm{Kc20me}$
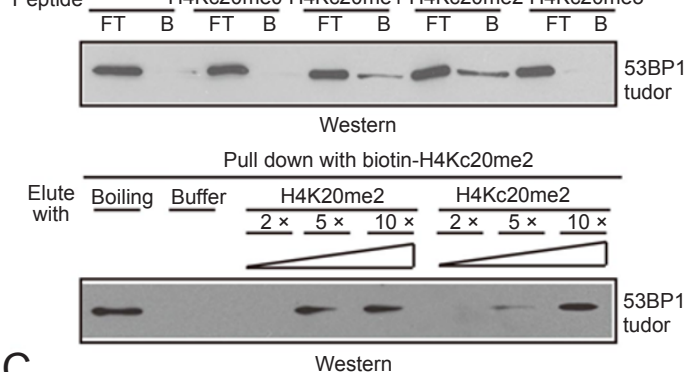

Peptide $\frac{-}{\mathrm{FT} B} \frac{\mathrm{H} 3 \mathrm{Kc9me}}{\mathrm{FT} B} \frac{\mathrm{H} 3 \mathrm{Kc9me}}{\mathrm{FT} \quad \mathrm{B}} \frac{\mathrm{H} 3 \mathrm{Kc9me}}{\mathrm{FT} \quad \mathrm{B}} \frac{\mathrm{H} 3 \mathrm{Kc9me}}{\mathrm{FT} B}$
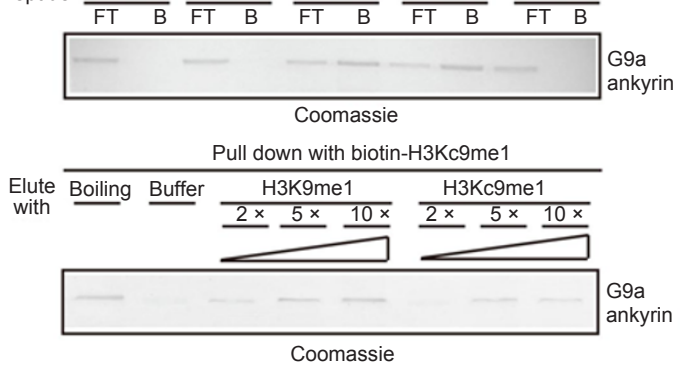

D
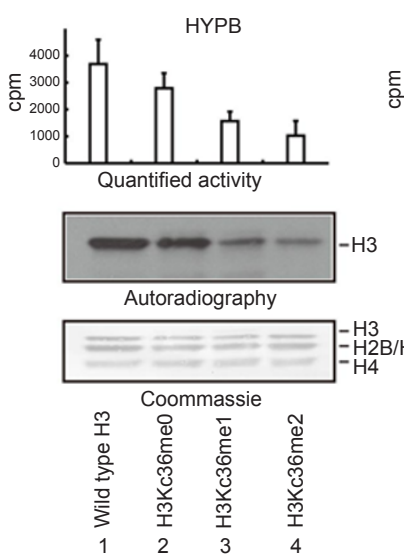

$\mathrm{F}$

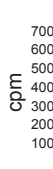

\begin{tabular}{r}
7000 \\
6000 \\
5000 \\
\hline \multicolumn{2}{c}{4000} \\
3000 \\
2000 \\
1000 \\
0
\end{tabular}
E
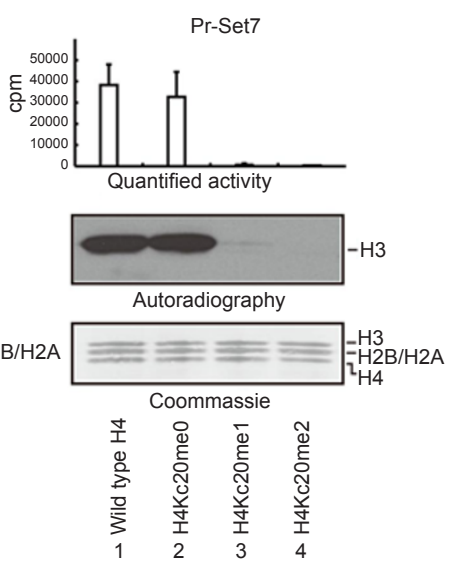

G
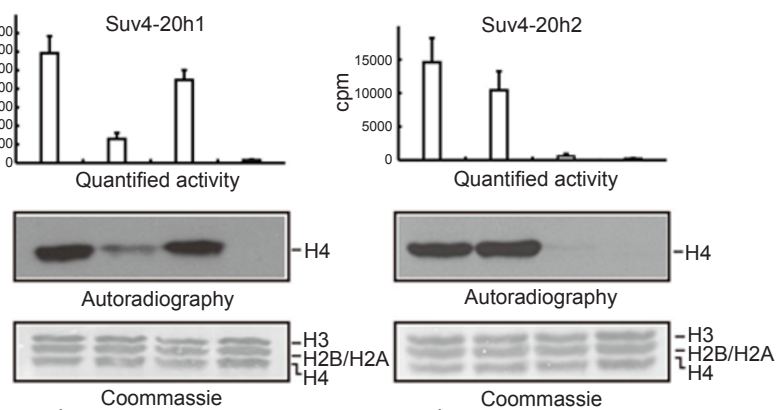
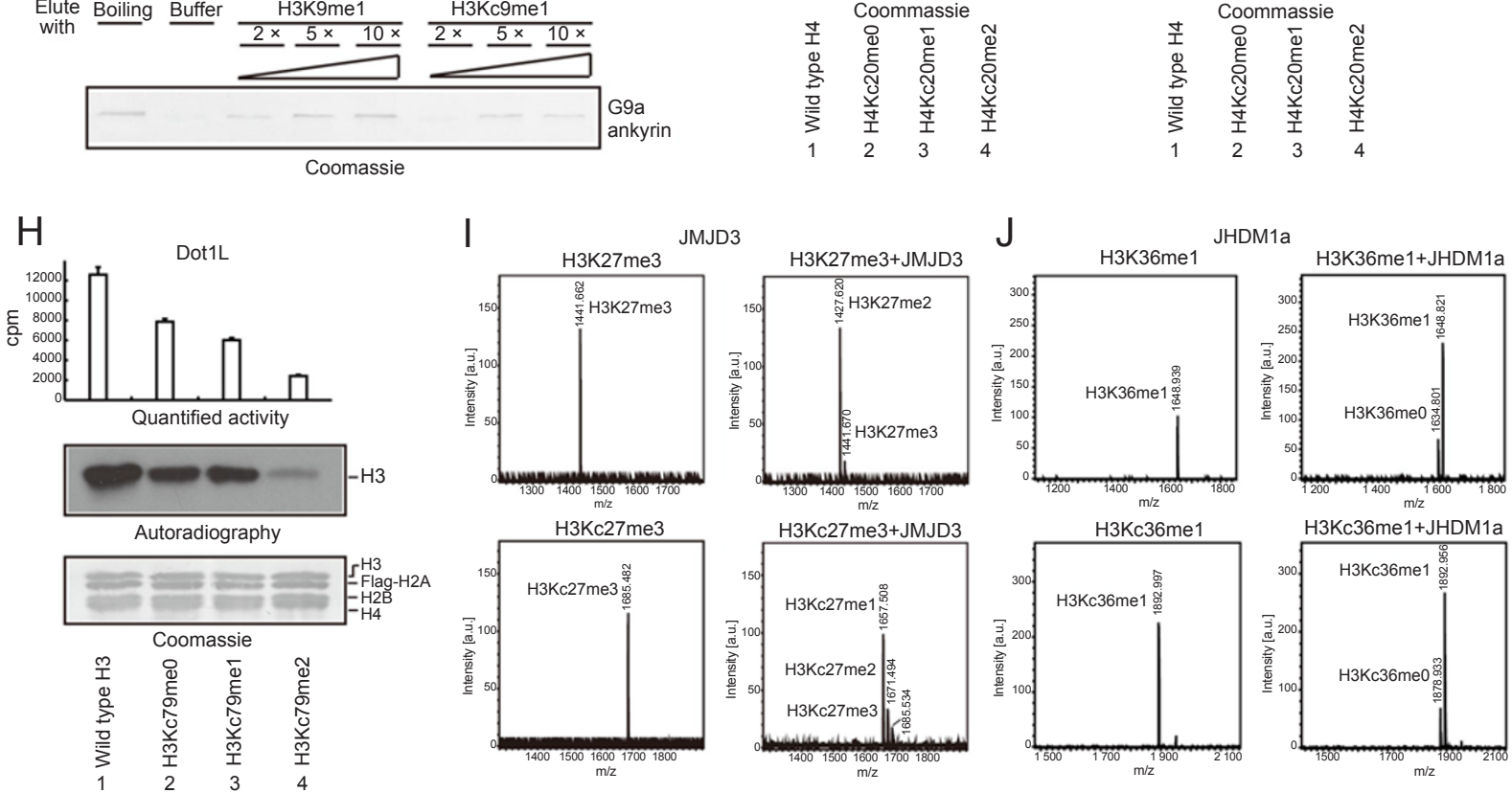

Cell Research | Vol 19 No 10 | October 2009 
regular $\mathrm{H} 3$ tail peptide [2]. Here, we analysed four different SET domain-containing HKMTs and one nonSET domain HKMT, Dot1L. Moreover, reconstituted nucleosomes were used as substrates instead of the histone tail peptides because many HKMTs are nucleosome-specific enzymes that do not react on histone peptides or core histones [1].

We first evaluated HYPB, an H3K36-specific HKMT. Recombinant HYPB SET domain displayed similar activity towards nucleosomes containing $\mathrm{H} 3 \mathrm{Kc} 36 \mathrm{me} 0$ (Figure 1D, lane 2) and nucleosomes containing wildtype H3 (Figure 1D, lane 1). This activity gradually declined when H3Kc36me1- and H3Kc36me2-containing nucleosomes were tested (Figure 1D, lanes 3-4), which is consistent with the fact that HYPB is a trimethylase $[5,6]$.

We then systematically analysed the three H4K20specific HKMTs, Pr-Set7 (also known as Set8), Suv420h1 and Suv4-20h2. In all three cases, MLA products were able to serve as substrates for the methylation reaction (Figure 1E-1G). Pr-Set7 displayed minimal activity towards H4Kc20me1 (Figure 1E, lane 3), consistent with the fact that Pr-Set7 is a mono-methylase [1]. To our surprise, despite the requirement of Suv420 family HKMTs for H4K20me3 in vivo [7], neither Suv4-20h1 nor Suv4-20h2 displayed detectable activity towards H4Kc20me2 (Figure 1F, lane 4; Figure 1G, lane 4). This is unlikely to be due to the MLA reaction, because both enzymes were capable of methylating $\mathrm{H} 4 \mathrm{Kc} 20 \mathrm{me} 0 / 1$ (Figure 1F, lanes 2-3; Figure 1G, lane 2 ). This implies that additional factor(s) might exist that regulate the methyl status specificity of Suv4-20 family HKMTs, especially that of Suv4-20h2, because it is the main enzyme responsible for maintaining H4K20me3 levels in vivo [7]. This is an intriguing hypothesis because, unlike the global distribution of H4K20me2 and the Suv4-20 family HKMTs [7], H4K20me3 is specifically enriched at pericentric heterochromatin [1], which suggests a requirement for a methyl status regulatory mechanism. Notably, the methyl status specificity of certain HKMT complexes has been shown to be regulated by regulatory subunits [8]. It is also worth noting that Suv4-20h1 displayed robust activity towards H4Kc20me1 (Figure 1F, lane 3), unlike Suv4-20h2 (Figure $1 \mathrm{G}$, lane 3). This implies that Suv4-20h1 may favour the H4K20me1 generated by Pr-Set7 as its substrate, whereas Suv4-20h2 may prefer to directly methylate unmethylated H4. Next, we tested Dot1L (the human homologue of yeast Dot1), which is the only known nonSET domain HKMT that specifically methylates H3K79 [1]. Once again, nucleosomes with MLAs could serve as substrates for Dot1L (Figure 1H).

In the previous section, we have shown the ability of HKMTs to add new methyl groups onto MLA products (Figures 1D-1H). Next, we assessed whether histone demethylases can remove the methyl groups from MLA products. JMJD3 is an H3K27-specific KDM that reacts with trimethylated substrate [9]. Incubation with recombinant JMJD3 effectively demethylated peptides containing $\mathrm{H} 3 \mathrm{~K} 27 \mathrm{me} 3$ (Figure 1I, upper panel) as well as MLA-generated H3Kc27me3 (Figure 1I, lower panel). In addition, the H3K36-specific demethylase JHDM1a [10] effectively demethylated H3K36me1 (Figure 1J, upper panel) and $\mathrm{H} 3 \mathrm{Kc} 36 \mathrm{mel}$ peptides (Figure $1 \mathrm{~J}$, lower panel). Our results collectively indicate that MLA products can serve as substrates for histone demethylases.

We have thus systematically evaluated the biochemical reaction compatibility of MLA products in different systems, including methylated histone-binding assays, histone methyltransferase assays and demethylase assays. The products of Shokat's reactions served as substrates for all the above biochemical reactions and retained

Figure 1 MLA products are compatible with various biochemical reactions. (A-C) MLA products are compatible with methylated histone-binding proteins. Upper panels, peptide pull-down assays. Lower panels, competition assays. FT: $10 \%$ of total flow through. B: $100 \%$ of bound material eluted from the magnetic streptavidin beads. (A) Recombinant HP1 binds MLA products with correct methyl status specificity. (B) Recombinant 53BP1 tudor domain binds the MLA products with correct methyl status specificity. (C) Recombinant G9a ankyrin repeats bind the MLA products with correct methyl status specificity. (D-H) MLAcontaining nucleosomes are compatible with various HKMTs. Bottom panels show the coomassie blue-stained membranes for nucleosome loading; middle panels are autoradiography results for the HKMT activity assay; upper panels are quantified data obtained by liquid scintillation counting, the standard deviations were obtained from three parallel experiments. (D) HYPB. (E) Pr-Set7. (F) Suv4-20h1. (G) Suv4-20h2. (H) Dot1L. (I-J) MLA products are compatible with histone demethylases. (I) MALDI-TOF mass spectrometry analysis of H3K27me3 peptide before (upper left panel) and after incubation with recombinant JMJD3 (upper right panel); MALDI-TOF mass spectrometry analysis of H3Kc27me3 peptide before (lower left panel) and after incubation with recombinant JMJD3 (lower right panel). (J) MALDI-TOF mass spectrometry analysis of H3K36me1 peptide before (upper left panel) and after incubation with recombinant JHDM1a (upper right panel); MALDI-TOF mass spectrometry analysis of H3Kc36me1 peptide before (lower left panel) and after incubation with recombinant JHDM1a (lower right panel). 
excellent substrate specificity in most cases, demonstrating their great potential as a powerful tool for histone lysine methylation studies. We hope that our results will encourage more researchers to incorporate Shokat's reaction into their studies.

\section{Acknowledgments}

The cDNA-encoding 53BP1 was kindly provided by Dr Lilin $\mathrm{Du}$ from the National Institute of Biological Sciences, Beijing. Recombinant JHDM1a was kindly provided by Dr Jijie Chai from the National Institute of Biological Sciences, Beijing. The cDNAencoding G9a was kindly provided by Dr Yoichi Shinkai from Kyoto University. This work was supported by the Chinese Ministry of Science and Technology 863 project 2007AA02Z1A6 to B.Z.

Guangshuai $\mathrm{Jia}^{1,2,3}$, Weixiang $\mathrm{Wang}^{3}$, Hong $\mathrm{Li}^{3}$, Zhuo $\mathrm{Mao}^{4,3}$, Gaihong $\mathrm{Cai}^{3}$, Jian $\mathrm{Sun}^{3}$, Hui $\mathrm{Wu}^{4,3}, \mathrm{Mo} \mathrm{Xu}^{4,3}$, Peng Yang ${ }^{5,3}$, Wen Yuan ${ }^{6,3}$, She Chen ${ }^{3}$, Bing Zhu ${ }^{3}$

${ }^{1}$ Immunobiology Laboratory and Overseas Team, Key Laboratory of Animal Model and Human Disease Mechanisms, Kunming Institute of Zoology, the Chinese Academy of Science, Kunming 650223, China; ${ }^{2}$ Graduate University of Chinese Academy of Science, Beijing 100049, China; ${ }^{3}$ National Institute of Biological Sciences, Beijing, 102206, China $;{ }^{4}$ Graduate Program, Peking Union Medical College and Chinese Academy of Medical Sciences, Beijing, 100730, China; ${ }^{5}$ College of Life Sciences, Peking University, Beijing 1000871, China; ${ }^{6}$ State Key Laboratory of Plant Physiology and Biochemistry, College of Biological Sciences, China Agricultural University, Beijing 100094, China

Correspondence: Bing Zhu

Tel: 86-10-80728458; Fax: 86-10-80715648

E-mail: zhubing@nibs.ac.cn

Abbreviations: MLA (methyl-lysine analogue); HKMT (histone lysine methyltransferase); KDM (Lysine demethylase); Kc ("pseudo lysine" or aminoethylcysteine); H3Kc9me2 (H3 with its amino acid No. 9 converted to N-dimethyl-aminoethylcysteine or "pseudo" dimethyl-lysine)

\section{References}

1 Allis CD, Jenuwein T, Reinberg D. In: Allis CD, Jenuwein T, Reinberg D, eds. Epigenetics. Cold Spring Harbor Laboratory Press: New York 2006:23-56.

2 Simon MD, Chu F, Racki LR, et al. The site-specific installation of methyl-lysine analogs into recombinant histones. Cell 2007; 128:1003-1012.

3 Botuyan MV, Lee J, Ward IM, et al. Structural basis for the methylation state-specific recognition of histone H4-K20 by 53BP1 and Crb2 in DNA repair. Cell 2006; 127:1361-1373.

4 Collins RE, Northrop JP, Horton JR, et al. The ankyrin repeats of G9a and GLP histone methyltransferases are mono- and dimethyllysine binding modules. Nat Struct Mol Biol 2008; 15:245-250.

5 Edmunds JW, Mahadevan LC, Clayton AL. Dynamic histone $\mathrm{H} 3$ methylation during gene induction: $\mathrm{HYPB} / \mathrm{Setd} 2$ mediates all H3K36 trimethylation. EMBO J 2008; 27:406-420.

6 Yuan W, Xie J, Long C, et al. Heterogeneous nuclear ribonucleoprotein $\mathrm{L}$ is a subunit of human KMT3a/Set2 complex required for $\mathrm{H} 3$ Lys-36 trimethylation activity in vivo. $J$ Biol Chem 2009; 284:15701-15707.

7 Schotta G, Sengupta R, Kubicek S, et al. A chromatin-wide transition to H4K20 monomethylation impairs genome integrity and programmed DNA rearrangements in the mouse. Genes Dev 2008; 22:2048-2061.

8 Schneider J, Wood A, Lee JS, et al. Molecular regulation of histone $\mathrm{H} 3$ trimethylation by COMPASS and the regulation of gene expression. Mol Cell 2005; 19: 849-856.

9 De Santa F, Totaro MG, Prosperini E, Notarbartolo S, Testa G, Natoli G. The histone H3 lysine-27 demethylase Jmjd3 links inflammation to inhibition of polycomb-mediated gene silencing. Cell 2007; 130:1083-1094.

10 Tsukada Y, Fang J, Erdjument-Bromage H, et al. Histone demethylation by a family of JmjC domain-containing proteins. Nature 2006; 439:811-816. 